\title{
DETERMINING GEOMETRIC PARAMETERS OF AGRICULTURAL TREES FROM LASER SCANNING DATA OBTAINED WITH UNMANNED AERIAL VEHICLE
}

\author{
E. Hadas ${ }^{1, *}$, G. Jozkow $^{1}$, A. Walicka ${ }^{1}$, A. Borkowski ${ }^{1}$ \\ ${ }^{1}$ Wrocław University of Environmental and Life Sciences, Institute of Geodesy and Geoinformatics, Grunwaldzka 53, 50-375 \\ Wrocław, Poland - (edyta.hadas, grzegorz.jozkow, agata.walicka, andrzej.borkowski)@upwr.edu.pl
}

\author{
Commission II, WG II/10
}

KEY WORDS: UAV, LiDAR, orchards, tree height, alpha-shape

\begin{abstract}
:
The estimation of dendrometric parameters has become an important issue for agriculture planning and for the efficient management of orchards. Airborne Laser Scanning (ALS) data is widely used in forestry and many algorithms for automatic estimation of dendrometric parameters of individual forest trees were developed. Unfortunately, due to significant differences between forest and fruit trees, some contradictions exist against adopting the achievements of forestry science to agricultural studies indiscriminately. In this study we present the methodology to identify individual trees in apple orchard and estimate heights of individual trees, using high-density LiDAR data $\left(3200\right.$ points $\left./ \mathrm{m}^{2}\right)$ obtained with Unmanned Aerial Vehicle (UAV) equipped with Velodyne HDL32-E sensor. The processing strategy combines the alpha-shape algorithm, principal component analysis (PCA) and detection of local minima. The alpha-shape algorithm is used to separate tree rows. In order to separate trees in a single row, we detect local minima on the canopy profile and slice polygons from alpha-shape results. We successfully separated $92 \%$ of trees in the test area. $6 \%$ of trees in orchard were not separated from each other and $2 \%$ were sliced into two polygons. The RMSE of tree heights determined from the point clouds compared to field measurements was equal to $0.09 \mathrm{~m}$, and the correlation coefficient was equal to 0.96 . The results confirm the usefulness of LiDAR data from UAV platform in orchard inventory.
\end{abstract}

\section{INTRODUCTION}

The estimation of dendrometric parameters has become an important issue for agriculture planning and for the efficient management of orchards (Kumar et al., 2008). Typically, the dendrometric parameters of trees, such as tree heights, crown base heights, stem and crown diameters are derived from direct field measurements (Brown, 1997), which are, however, time consuming, inefficient, and expensive. Remote sensing technologies, such as the laser scanning, can be used to collect data for the large areas within a relatively short time. However, the point clouds obtained from LiDAR (Light Detection and Ranging) need a proper processing in order to estimate the geometric parameters of trees. Nowadays, there exist many algorithms that are designed for an automatic estimation of geometric parameters of forest trees (Hyyppä et al., 2012). Unfortunately, there is a limited number of algorithms that were tested for trees of a special interest, such as agriculture trees in orchards. Recio et al. (2013) used k-means algorithm followed by an automatic detection of the classes representing trees in order to detect fruit trees and extract selected trees and plot-based parameters. Estornell et al. (2014) elaborated an area based approach for the tree height and biomass estimation of olive trees, using sparse ALS data $\left(0.5\right.$ points $\left./ \mathrm{m}^{2}\right)$. Hadas et al. (2017) proposed a method that combines alpha-shape algorithm with principal component analysis to estimate geometric parameters of individual trees in olive orchards. Tree parameters such as tree diameter, tree height, crown area, are the most widely applied predictors of biomass, when combined with an appropriate and reliable allometric model (Kuyah and Rosenstock, 2014).

One has to note that there are many differences between forest and fruit trees, e.g. a biomass of fruit tree is concentrated in the crown, not in a stem, that is the case for forest trees, therefore the characteristics of a crown is of particular interest
(Velázquez-Martí et al., 2010). Human management, such as pruning and fertilization, as well as heterogeneous growing conditions can change crown architecture (Harja et al. 2012). This causes some contraindications against adopting the achievements of forestry science to agricultural studies indiscriminately.

Finally, from economical point of view, for orchard areas it is reasonable to use unmanned aerial vehicle (UAV) instead of a plane or a helicopter. UAV derived laser scanning data has different specific from classical ALS data. UAV LiDAR uses smaller and lighter sensors that have typically lower performance, resulting in a lower accuracy of the point cloud. On the other hand, UAV can fly relatively low and slow resulting in much denser point clouds. In some orchard areas it can even operate between trees, that allows to obtain not only top but also side reflections from trees, since many LiDAR UASs (Unmanned Aerial Systems) have very wide field of view or allow to mount LiDAR sensor for side scanning. The accuracy of point cloud collected with small UASs can be better than $10 \mathrm{~cm}$, thus fits mapping-grade category (Jozkow et al., 2017).

In this study we present the original methodology to identify individual trees in apple orchard and to estimate heights of individual trees from high-density LiDAR data obtained with UAV. We compare the results of LiDAR data processing with direct field measurements in order to validate the method and identify directions of further studies.

\section{DATA AND METHODS}

\subsection{Test area}

The test area is a part of an orchard located near Trzebnica city, dolnośląskie voivodeship, South West Poland. The test area is

\footnotetext{
* Corresponding author
} 
about $10000 \mathrm{~m}^{2}$ large, and is located on a mild slope ( 5\%). It is covered by over 600 apple trees (malus domestica 'Idared'), planted in 26 rows.

During the measurement campaign, trees were leafy and had ripe fruits (Figure 1). The ground between rows was covered with long grass and some nettle. Around tree stems some pesticides were applied resulting in low ground vegetation.

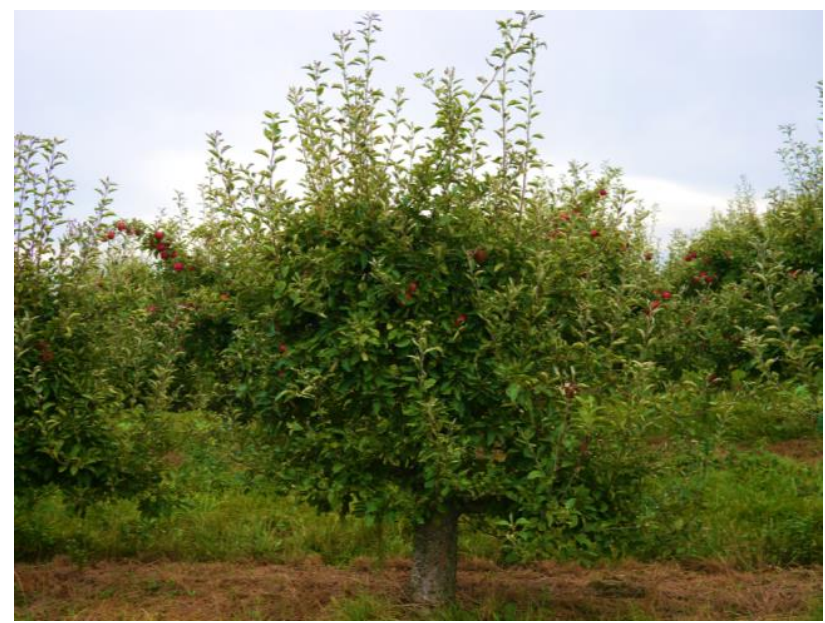

Figure 1. An apple tree in the test orchard

\subsection{LiDAR data}

In order to obtain LiDAR data for the test area, small Unmanned Aerial System (Figure 2) was used: Aibot X6 V2 hexacopter (Karpina et al. 2016), equipped with Velodyne HDL32-E laser scanner, geodetic-grade dual frequency Global Navigation Satellite Systems (GNSS) receiver, and tacticalgrade Inertial Measurement Unit (IMU).

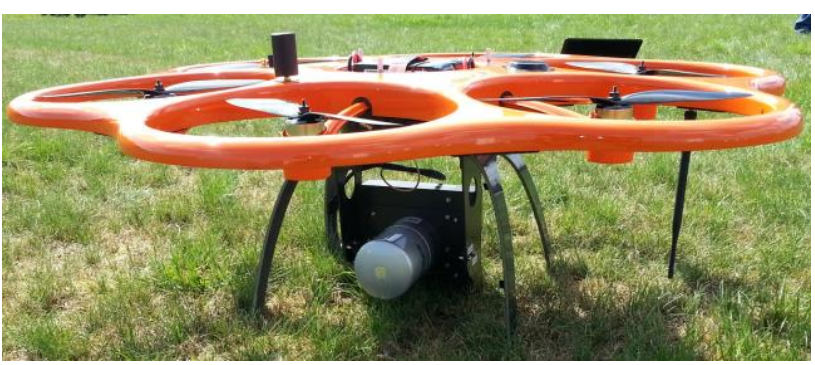

Figure 2. Unmanned Aerial System used to collect LiDAR data

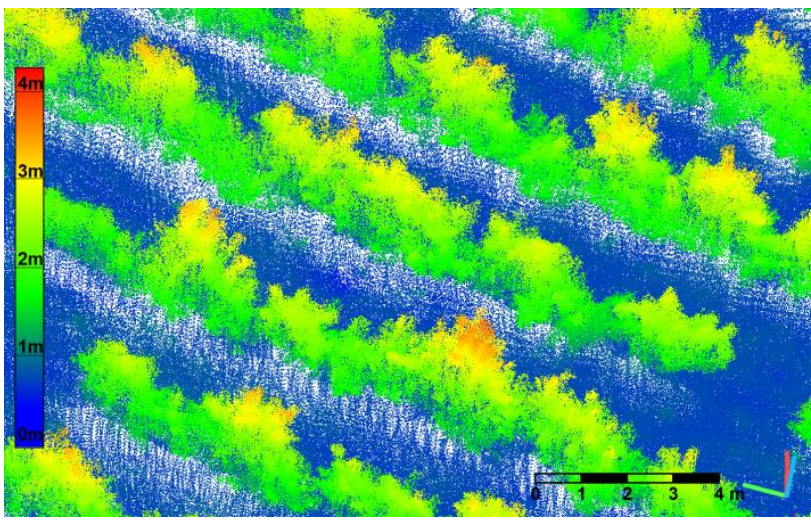

Figure 3. Plot of the point cloud obtained for a part of the study area; height coded with colour
The measurement campaign took place on September 26, 2017. The average flight height was equal to $30 \mathrm{~m}$. We obtained about 32 million of points in the orchard, that results in average point cloud density of about 3200 points $/ \mathrm{m}^{2}$. A sample of collected point cloud is shown in Figure 3.

\subsection{Reference data}

The reference data was collected in September 2017. 50 trees in the orchard were selected, for which reference measurements were obtained by means of GNSS and ruler. GNSS receiver was operating in Real Time Network (RTN) mode, receiving RTN corrections from NAWGEO service of ASG-EUPOS network (Bosy et al., 2007). Such configuration ensured the horizontal and vertical accuracies of about $3 \mathrm{~cm}$ and $5 \mathrm{~cm}$, respectively. We measured 3D coordinates of tree top and stem bottom, and also delineated crown contours. With a ruler we measured crown base height relative to the ground and stem circumference just below the crown. These measurements allowed to determine tree height, crown diameters and stem diameter.

Moreover, ortophotomap from ISOK (IT System of the Country's Protection against extreme hazards), obtained in 2014 with a ground sample distance (GSD) equal to $0.1 \mathrm{~m}$ was used, and all trees in the test area were manually digitized as points. We tried to locate a point in a central part of the crown, hence we call them tree centroids further in this paper. Tree centroids from ortophotomap were visually compared with the point cloud in order to correct commission and omission errors caused by misinterpretation of the ortophotomap and due to a few tree cut-outs over the last 3 years.

\subsection{Processing strategy}

The data collected by navigation sensors and laser scanner was used in order to get the georeferenced point cloud. A typical workflow for the ALS data was used. It consists of three main steps. In the first step GNSS differential observations (from the ground base station and drone rover receiver) and IMU are integrated in order to get the 6 degree of freedom trajectory (position and orientation) of the UAV. The position and orientation of the UAV are used in the second step to transform coordinates of points from local scanner coordinate system into global coordinate system. In the last step the transformation from global coordinate system into national coordinate system is applied.

The open-source Cloud Compare software was used to automatically classify point clouds (ground and vegetation) using Cloth Simulation Filter (Zhang et al., 2016). The Digital Terrain Model (DTM) with a grid size of $0.5 \times 0.5 \mathrm{~m}$ was generated using ground points. Then the distance between offground points from the DTM was calculated. The original point heights were replaced by the distances from DTM in order to obtain the normalized point cloud of the apple trees.

In the next step alpha-shape algorithm (Edelsbrunner et al., 1983) was applied to the normalized point cloud in order to delineate crowns. For the alpha shape algorithm we defined the radius $\mathrm{R}=0.5 \mathrm{~m}$. $\mathrm{R}$ characterizes the largest empty circle containing the edge or triangle of Delaunay triangulation. Moreover, points with relative heights below $0.8 \mathrm{~m}$ were not considered, in order to remove low vegetation and possible DTM errors. However, due to dense plantation of trees and limited pruning of low branches, tree crowns in a row overlapped each other. Therefore, as a results of alpha-shape, 
we obtained contours that usually contain more than one single tree. In order to separate tree rows into single trees, Principal Component Analysis (PCA) was performed on planar coordinates of the point cloud inside each row contour. Then, for each row separately, normalized heights and the first component of PCA were used to generate a canopy profile with a ground sampling equal to $0.1 \mathrm{~m}$. Then the row contour was split by lines crossing the local minima identified along the profile. For each detected tree contour (region between two crossing lines), a tree height was determined as a height of the highest points inside the tree contour.

\subsection{Validation}

First, tree identification results in the entire test area were checked. The assumption is that a tree is identified correctly if its crown contour contains only one tree centroid. Therefore, the comparison of contours and centroids may result in two types of identification errors: 1) omission - when two or more trees are not separated from each other, i.e. there are more than one tree centroid inside a crown contour and 2) commission - when a single tree is represented by more than one contour, i.e. there is a polygon without any centroid inside. Secondly, the accuracy of tree height determination was analysed by comparing heights obtained from LiDAR data to field measurements of the 50 reference trees.

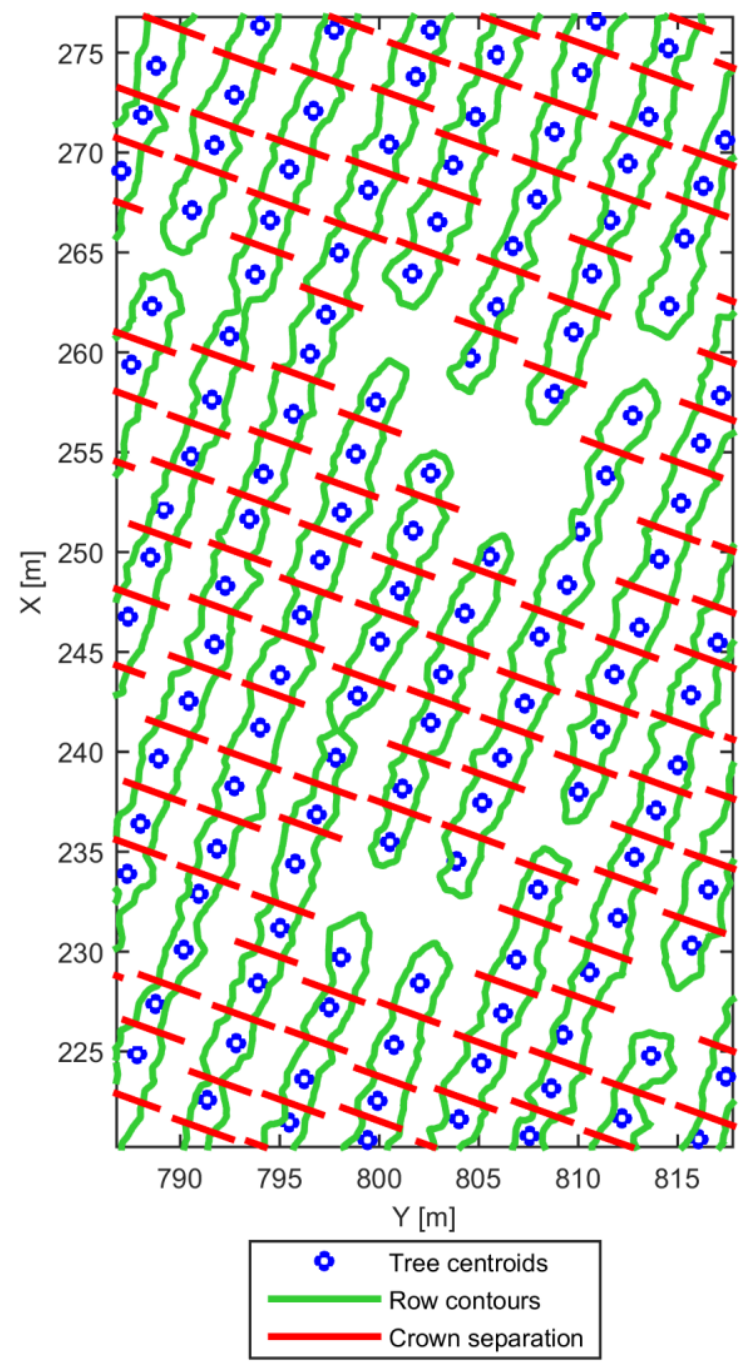

Figure 4. Result of tree crown delineation for a part of study area

\section{RESULTS}

\subsection{Tree identification}

The results of tree crown delineation are presented in Figure 4. There were 655 apple trees in the test area. 603 trees were correctly identified and separated from other trees, which gave the success rate of 92\%. 21 omission errors were noticed: 20 cases when two trees are represented by a single polygon, and one case when three trees are joined. This gave 43 trees in total that were not detected correctly. There was no case, when trees from different rows were joined, because there were large distances between tree rows. 10 commission errors were noticed, which occur only on row margins and in case when there were a cut-out trees inside a row.

Results showed that further improvements in crown delineation are required. Tree separation can possibly be improved by adding a constraints of local minima detection algorithm: minimum distance between consecutive minima and depression size. Alternatively, one can analyse the ratio of length and width of all detected crowns and force the split of outlying objects. In order to avoid commission error on a row margin, one can remove local minima that are too close to the margin. However, the definition of such a threshold requires a priori knowledge on typical size of crown contours in the test area.

\subsection{Tree height}

All 50 reference trees were identified and separated correctly from other trees. Therefore tree heights obtained from LiDAR data were compared with heights measured directly in the field with GNSS RTN technique for all reference trees (Figure 5).

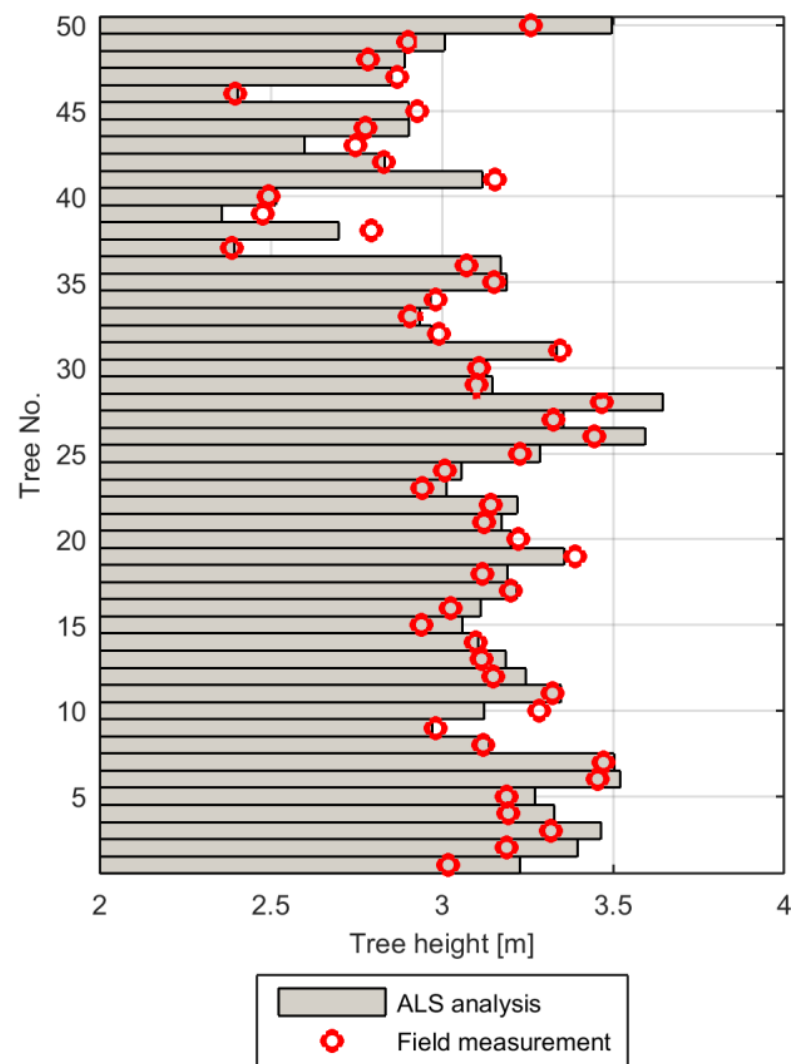

Figure 5. Comparison of tree heights obtained from LiDAR data analysis and field measurements for 50 reference trees 
We noticed good agreement between heights obtained from UAV LiDAR data and values measured in the field. The RMSE of tree height determined from LiDAR data was equal to $0.09 \mathrm{~m}$, while the correlation coefficient between this and reference heights was equal to 0.96 . The maximum absolute error reached $0.24 \mathrm{~m}$. Surprisingly, most of the tree heights obtained from LiDAR data were slightly overestimated in comparison to field measurements. The mean difference was equal to $+0.04 \mathrm{~m}$. This is not a typical case (Hadas et al., 2016) and we explain this by the low quality of DTM below the trees. Moreover, trees are not rigid objects, and the highest branch swings easily on the wind.

\section{CONCLUSIONS}

We demonstrate how to obtain and process high density LiDAR data collected with UAS in order to identify trees in the orchard and determine tree heights. The processing strategy requires to normalize the point cloud, by calculating point distances from digital terrain model. The LiDAR data processing strategy combines alpha-shape algorithm, PCA and detection of local minima. We determine tree height as a height of the highest point inside the crown contour.

The proposed method of tree crown identification allows to successfully separate $92 \%$ of trees. 43 trees were not separated correctly from each other, and there were 10 commission errors. We determined tree heights with RMSE $=0.09 \mathrm{~m}$. The correlation coefficient between tree heights determined from LiDAR data and field measurements was equal to 0.96. The results confirm the usefulness of LiDAR data collected with small UAS in orchard inventory.

The presented research are part of undertaken studies on automatic estimation of geometric parameters of trees in orchards. The following studies will also concern a determination of crown contours, crown diameters and crown base heights. This will require further improvements in the proposed methodology. The impact of UAV LiDAR data density on the results by subsampling the original point cloud will also be investigated. The methodology will be validated on two other test areas: peach and cherry orchards. We will try to provide a set of optimum LiDAR data processing parameters depending on the orchard characteristics.

\section{ACKNOWLEDGEMENTS}

This work has been supported by the National Science Centre, Poland, research Project No. UMO-2016/23/N/ST10/00180 and the Wroclaw Center of Networking and Supercomputing (http://www.wcss.wroc.pl/) computational Grant using MATLAB Software License No.: 101979.

\section{REFERENCES}

Bosy, J., Graszka W., Leończyk M., 2007. ASG-EUPOS. A Multifunctional Precise Satellite Positioning System in Poland. European Journal of Navigation, 5(4), pp. 2-6.

Brown, S., 1997. Estimating biomass and biomass change of tropical forests: a primer. FAO Forestry Paper-134, Food and Agriculture Organization of the United Nations (FAO), Rome

Edelsbrunner, H., Kirkpatrick, D.G., and Seidel R., 1983. On the shape of a set of points in the plane. IEEE Transactions on Information Theory, 29(4), pp. 551-559

https://doi.org/10.1109/TIT.1983.1056714
Estornell, J., Velázquez-Martí, B., López-Cortés, I., Salazar, D., and Fernández-Sarría, A., 2014. Estimation of wood volume and height of olive tree plantations using airborne discretereturn LiDAR data. GISci. Remote Sens., 51 (1), pp. 17-29 https://doi.org/10.1080/15481603.2014.883209

Hadas, E., Borkowski, A., Estornell, J., and Tymków, P., 2017. Automatic estimation of olive tree dendrometric parameters based on airborne laser scanning data using alpha-shape and principal component analysis. GISci. Remote Sens., 54(6), pp. 898-917

https://doi.org/10.1080/15481603.2017.1351148

Hadas, E., Estornell J., 2016. Accuracy of tree geometric parameters depending on the LiDAR data density. European Journal of Remote Sensing, 49, pp. 73-92 https://doi.org/10.5721/EuJRS20164905

Harja, D., Vincent, G., Mulia, R., and Van Noordwijk, M., 2012. Tree shape plasticity in relation to crown exposure. Trees, 26, pp.1275-1285

https://doi.org/10.1007/s00468-012-0703-x

Hyyppä, J., Holopainen M., and Olsson H., 2012. Laser Scanning in Forests. Remote Sensing, 4(10), pp. 2919-2922 https://doi.org/10.3390/rs4102919

Jozkow G., Wieczorek P., Karpina M., Walicka A., Borkowski A., 2017. Performance Evaluation of sUAS Equipped with Velodyne HDL-32E Lidar Sensor. Int. Arch. Photogramm. Remote Sens. Spatial Inf. Sci., XLII-2/W6, pp. 171-177 https://doi.org/10.5194/isprs-archives-XLII-2-W6-171-2017

Karpina, M., Jarząbek-Rychard, M., Tymków, P., Borkowski, A., 2016. UAV-based automatic tree growth measurement for biomass estimation. In: ISPRS Annals of the Photogrammetry, Remote Sensing and Spatial Information Sciences, VIII-7/W7, pp. 685-688.

http://doi.org/10.5194/isprsarchives-XLI-B8-685-2016

Kumar, A., Singh, K.N., Lal, B., and Singh, R.D., 2008. Mapping of apple orchards using remote sensing techniques in cold desert of Himachal Pradesh, India. Remote Sensing, 36(4), pp. 387-392

https://doi.org/10.1007/s12524-008-0038-7

Kuyah, S., and Rosenstock, T.S., 2015. Optimal measurement strategies for aboveground tree biomass in agricultural landscapes. Agroforest Syst., 89(1), pp. 125-133

https://doi.org/10.1007/s10457-014-9747-9

Recio, J.A., Hermosilla, T., Ruiz, L.A., and Palomar J., 2013. Automated extraction of tree and plot-based parameters in citrus orchards from aerial images. Comput. Electron. Agric., 90, pp. 24-34

https://doi.org/10.1016/j.compag.2012.10.005

Velázquez-Martí, B., Fernández-González, E., Estornell, J., and Ruiz, L.A., 2010. Dendrometric and dasometric analysis of the bushy biomass in Mediterranean forests. For. Ecol. Manage., 259 (5), pp. 875-882

https://doi.org/10.1016/j.foreco.2009.11.027

Zhang, W., Qi, J., Wan, P., Wang, H., Xie, D., Wang, X., and Yan, G., 2016. An Easy-to-Use Airborne LiDAR Data Filtering Method Based on Cloth Simulation. Remote Sensing, 8(6), p. 501

https://doi.org/10.3390/rs8060501 